\title{
Magnetic fluctuations in coupled inequivalent Hubbard layers as a model for $\mathrm{Y}_{2} \mathrm{Ba}_{4} \mathrm{Cu}_{7} \mathrm{O}_{15}$
}

\author{
G. Hildebrand ${ }^{1}$, E. Arrigoni ${ }^{1}$, J. Schmalian ${ }^{2}$, and W. Hanke ${ }^{1}$ \\ ${ }^{1}$ Institut für Theoretische Physik, Universität Würzburg, Am Hubland, D-97074 Würzburg, Germany \\ ${ }^{2}$ Department of Physics, University of Illinois at Urbana-Champaign, $1110 \mathrm{~W}$. Green Str., Urbana 61801, IL
}

\begin{abstract}
We investigate, within the fluctuation-exchange approximation, a correlated-electron model for $\mathrm{Y}_{2} \mathrm{Ba}_{4} \mathrm{Cu}_{7} \mathrm{O}_{15}$ represented by two inequivalent Hubbard layers coupled by an interlayer hopping $t_{\perp}$. An energy offset $\delta$ is introduced in order to produce a different charge carrier concentration in the two layers. We compare several single-particle and magnetic excitations, namely, the single particle scattering rate, the spectral function and the spin lattice as well as spin-spin relaxation times in the two layers as a function of $\delta$. We show that the induced interlayer magnetic coupling produces a tendency to "equalization" of the magnetic properties in the two layers whereby antiferromagnetic fluctuations are suppressed in the less doped layer and enhanced in the heavily doped one. The strong antiferromagnetic bilayer coupling causes the charge carriers in the plane with larger doping concentration to behave similar to those of the underdoped layer, they are coupled to. This effect grows for decreasing temperature. For high temperatures or if both layers are optimally or overdoped, i.e. when the antiferromagnetic correlation length becomes of the order or smaller than one lattice site the charge carrier and magnetic dynamics of the two layers is disconnected and the equalization effect disappears. These results are in good agreement with NMR experiments on $\mathrm{Y}_{2} \mathrm{Ba}_{4} \mathrm{Cu}_{7} \mathrm{O}_{15}$ by Stern et al. Phys. Rev B 51, 15478 (1995). We also compare the results with calculations on bilayer systems with equivalent layers as models for the constituent compounds $\mathrm{YBa}_{2} \mathrm{Cu}_{3} \mathrm{O}_{7}$ and $\mathrm{YBa}_{2} \mathrm{Cu}_{4} \mathrm{O}_{8}$.
\end{abstract}

PACS numbers: 74.72.-h,71.27.+a,76.60.-k

\section{INTRODUCTION}

There is a large amount of consensus that the anomalous properties of cuprate superconductors are caused by the strongly interacting electrons within the $\mathrm{CuO}_{2}$ planes. However, in particular the observation that the highest superconducting transition temperatures belong to compounds with more than one layer per unit cell initiated various investigations of out-of-plane properties. The observation of a rather strong coupling between adjacent layers has been made by inelastic neptron scatteringl (INS), nuclear magnetic resonance 35 (NMR) and indirectly also in Raman scattering experiments 6 . Furthermore, the observation of a qualitatively different behavior of the odd and even channel in INS including a sharp resonance feature, found solely for odd excitations and of a bilayer splitting of the Fermi surface found in angular resolved photoemission experiments (ARPES) 8 . demonstrate that low energy excitations of cuprates are affected by the presence of more than one layer per unit cell. Related to these issues is the interesting question of the c-axis transport and the occurrence of a c-axis Josephson plasma excitation 10,11 , which may turn out to be a new probe of the vortex statics and dynamics of the superconducting state.

A very interesting perspective on the nature of the coupling between $\mathrm{CuO}_{2}$-layers was offered by NMR experiments by Stern et al. on $\mathrm{Y}_{2} \mathrm{Ba}_{4} \mathrm{Cu}_{7} \mathrm{O}_{15}$ (247). This material has a variety of structural similarities to the extensively studied $\mathrm{YBa}_{2} \mathrm{Cu}_{3} \mathrm{O}_{7}$ (123) and $\mathrm{YBa}_{2} \mathrm{Cu}_{4} \mathrm{O}_{8}$ (124) systems. The main difference in the crystallographic structure of 123 and 124 is the double $\mathrm{CuO}$ chain in the latter. The compound 247 can be considered as a natural multilattice, consisting of alternating 124 and 123 blocks. The bilayers in 247 are correspondingly build up of one $\mathrm{CuO}_{2}$ layer which belongs to the 123 block and one layer of the 124 block. Based on the analysis of the NQR spectra it turned out that the charge carrier content in these nonequivalent adjacent layers is very close to that of the related parent compounds of the two blocks, i.e. one plane has a similar charge carrier concentration to the slightly overdoped 123 system whereas the other layer corresponds to the underdoped 124 system. Interestingly, the highest transition temperature $\left(\mathrm{T}_{c}=95 \mathrm{~K}\right)$ is for 247 , which has to be compared with the $92 \mathrm{~K}$ for 123 and $82 \mathrm{~K}$ of the 124 system. The main experimental observations of Ref. 2.,3 are the following: (i) the lowtemperature Knight-shift suppression for both planes is, despite their different charge carrier concentration, similar and behaves like in the underdoped 124 system, even though the high temperature values of the Knight shift are the same as in the corresponding 123 and 124 compounds. (ii) both ${ }^{63} \mathrm{Cu}$ spin lattice relaxation times of the two different planar $\mathrm{Cu}$-sites show a spin pseudogap in $1 /{ }^{63} T_{1} T$, even though it is barely present in the 123 parent compound itself. (iii) the interplane transverse relaxation rate, as measured in a spin-echo double resonance experiment (which characterizes the interplane magnetic susceptibility) increases for decreasing temperature faster than the intraplane relaxation rate. Thus, the main con- 
clusions from these observations are that, for high temperatures, the two planes are rather disconnected and behave similarly to their parent compounds, whereas for decreasing temperatures, the increasing interlayer magnetic coupling enforces even the slightly overdoped plane to behave like an underdoped system.

For a proper interpretation of these interesting experimental data and, in a more broader context, for a better understanding of the bilayer coupling in cuprate superconductors in general, it is essential to investigate to what extent one can describe the main trends of these data within a model of coupled layers, only different by their charge carrier concentration or whether one needs to make qualitatively new assumptions about the nature of the bilayer coupling.

One promising approach for the description of bilayer phenomena is based on a Hubbard Hamiltonian with local repulsive Coulomb interaction, where the interplanar coupling is caused solely by an interplane hopping element $t_{\perp}$. This model, restricted to the case of equivalent layers $(\delta-0)$, has been investigated within various technique 12 14. Additional insight can be gained using a self-consistent summation of bubble and ladder diagrams (fluctuation exchange pproximation). The main results of these investigation 1518 are enhanced antiferromagnetic spin fluctuations due to layer coupling causing, in bilayer systems as well, a $\mathrm{d}_{x^{2}-y^{2}}$ symmetry of the superconducting order parameter, a predominantly incoherent low energy c-axis charge transport even though the bilayer splitting stays intact, and an enhancement of the relative strength of interlayer vs. intralayer coupling for decreasing doping.

In this paper, we additionally consider the effect of an energy offset $\delta$, which produces a different charge carrier concentration in the two Hubbard layers (cf. also Scalettar et al.19). This is a suitable model to describe the peculiarity of the 247 compound $\mathrm{Y}_{2} \mathrm{Ba}_{4} \mathrm{Cu}_{7} \mathrm{O}_{15}$, whose bilayers are build up of one $\mathrm{CuO}_{2}$ layer belonging to an $\mathrm{YBa}_{2} \mathrm{Cu}_{3} \mathrm{O}_{7}$ block and one to a $\mathrm{YBa}_{2} \mathrm{Cu}_{4} \mathrm{O}_{8}$ block. We evaluate several single-particle and magnetic excitations, namely, the single-particle scattering rate, the spectral function and the spin lattice as well as spin-spin relaxation times in the two inequivalent layers as a function of $\delta$ within the fluctuation-exchange approximation. We show that the interlayer coupling produces a tendency to equalization of the antiferromagnetic properties in the two layers whereby antiferromagnetic fluctuations are suppressed in the less doped layer and enhanced in the heavily doped one. This equalization effect turns out to be enhanced in the presence of antiferromagnetic fluctuations in the system and to be almost absent when the antiferromagnetic correlation length becomes of the order or smaller than one lattice site and to ultimately decrease for increasing temperature. These results are in good qualitative agreement with NMR experiments on $\mathrm{Y}_{2} \mathrm{Ba}_{4} \mathrm{Cu}_{7} \mathrm{O}_{15}$ by Stern et al.2.1. We also compare the results with calculations on bilayer systems with equivalent layers as models for the constituent compounds
$\mathrm{YBa}_{2} \mathrm{Cu}_{3} \mathrm{O}_{7}$ and $\mathrm{YBa}_{2} \mathrm{Cu}_{4} \mathrm{O}_{8}$.

A first theoretical investigation of the experimental findings of Ref. 3 has been given by Millis and Monien20, who could determine the size of the interlayer exchange coupling from an analysis of the interlayer cross relaxation time. These authors also discuss that the $41 \mathrm{meV}$ excitation observed in superconducting $\mathrm{YBa}_{2} \mathrm{Cu}_{3} \mathrm{O}_{7}$ is a collective mode pulled down below the superconducting gap by interactions, and that the observed antisymmetry under interchange of planes follows from the nonnegligible value of $J_{\perp}$. An analysis of the coupling between an undoped layer and an underdoped one, similar in spirit to ours, has been carried out by Scalettar et al.19. These authors study the pairing mechanism, which arises from the coupling of holes in doped layers to spin fluctuations in the undoped layers in analogy with the Ginzburg-type scenario for the coupling of electrons through excitons in a doped semiconductor. However, it turns out that magnetic fluctuations in the undoped layer are strongly suppressed by the coupling with the doped layer and superconducting correlations are reduced by the interplane coupling at least at the temperatures accessible to the simulations. The study of the coupling between a strongly antiferromagnetic and a doped subsystem has some similarities with the "stripe scenario" where hole-poor antiferromagnetic regions are considered to be in contact with hole-rich superconducting regions.

The paper is organized as follows: In Sec. II we present our model for coupled layers with different charge carrier concentration and summarize the main concept of the fluctuation exchange approximation, used for the approximate investigation of the model. In Sec. III] we present our numerical results with particular emphasis to the single-particle and magnetic fluctuations in the two layers and focus on the anisotropy and on the tendency of equalization of this effects. In order to make contact with the experimental investigations on the 247 system, we discuss at length the temperature dependence of various NMR quantities in Sec. [V]. Finally our results are summarized in Sec. V.

\section{MODEL AND TECHNIQUE}

In order to describe the strong electronic correlations in the high- $T_{c}$ superconductors and the particularities of the system $\mathrm{Y}_{2} \mathrm{Ba}_{4} \mathrm{Cu}_{7} \mathrm{O}_{15}$, consisting of two layers with different charge carrier concentration, we use a system of two two-dimensional Hubbard layers coupled by an hopping element $t_{\perp}$. After Fourier transformation of the intraplane sites into momentum space with in-plane momentum $\mathbf{k}$, the Hamiltonian reads:

$$
H=\sum_{\substack{l_{1}, l_{2} \\ \mathbf{k}, \sigma}}\left[H_{\mathrm{o}}(\mathbf{k})\right]_{l_{1}, l_{2}} c_{\mathbf{k}, l_{1}, \sigma}^{\dagger} c_{\mathbf{k}, l_{2}, \sigma}+U \sum_{i} n_{i, \uparrow} n_{i, \downarrow}
$$

where $c_{\mathbf{k}, l_{1}, \sigma}^{\dagger}$ creates a particle with spin $\sigma$ at momentum $\mathbf{k}$ in layer $l_{1}$. Furthermore, $n_{i, \uparrow}$ is the density operator 
at lattice site $\mathbf{R}_{i}$ and spin $\uparrow$, and $H_{\mathrm{o}}(\mathbf{k})$ the Hamilton matrix for the noninteracting system

$$
H_{\mathrm{o}}(\mathbf{k})=\left(\begin{array}{cc}
\epsilon_{\mathbf{k}}-\mu & t_{\perp} \\
t_{\perp} & \epsilon_{\mathbf{k}}+\delta-\mu
\end{array}\right) .
$$

In order to describe theoretically a different charge carrier concentration in the two layers, we additionaly introduce an on-site energy $\delta$ in the second layer, effectively modifying its chemical potential. The planes are coupled solely through a bare interplane hopping $t_{\perp}$. Furthermore, the bare energy dispersion in each plane is

$$
\begin{aligned}
\epsilon_{\mathbf{k}}= & -2 t\left(\cos k_{x}+\cos k_{y}\right)-4 t^{\prime} \cos k_{x} \cos k_{y} \\
& -2 t^{\prime \prime}\left[\cos \left(2 k_{x}\right)+\cos \left(2 k_{y}\right)\right] .
\end{aligned}
$$

thus including second and third-neighbor hopping processes $\left(t^{\prime}, t^{\prime \prime}\right)$ to better model the Fermi surface for the system under consideration. In the following calculation, we always set $t_{\perp} / t=0.4, U=4 t_{12}^{12}, 18$ and measure the energies in units of the next-nearest neighbor hopping $t$.

A diagonalization of $H_{\mathrm{o}}(\mathbf{k})$ leads to the bonding and anti-bonding bands of the noninteracting system

$$
\epsilon_{\mathbf{k}}^{ \pm}=\epsilon_{\mathbf{k}}+\frac{\delta}{2} \pm \sqrt{\frac{\delta^{2}}{4}+t_{\perp}^{2}} .
$$

The single-particle excitations and the thermodynamic properties of the interacting system are deduced from the Green's function $G\left(\mathbf{k}, i \omega_{\mathrm{m}}\right)$ obtained through Dyson's equation which for a two-layer system generalizes to a $(2 \times 2)$ matrix equation

$$
G^{-1}\left(\mathbf{k}, i \omega_{\mathrm{m}}\right)=\left(i \omega_{\mathrm{m}}+\mu\right) \mathbf{1}-H_{\mathrm{o}}(\mathbf{k})-\Sigma\left(\mathbf{k}, i \omega_{\mathrm{m}}\right) .
$$

Approximations are introduced by the explicit choice of the self-energy $\Sigma\left(\mathbf{k}, i \omega_{\mathrm{m}}\right)$. Here, we use the expression for the self-energy given by the FLEX approximation 21 without particle-particle vertex contributions. Within the FLEX, the irreducible particle-particle vertex is solely the repulsive Coulomb interaction $U$ and consequently irrelevant. Interference effects between the particleparticle and particle-hole channel, which may be of relevance for an understanding of the pseudogap state of underdoped cuprates at low temperatures, are beyond the scope of this paper.

Introducing the shorthand notation $k \equiv\left(\mathbf{k}, i \omega_{\mathrm{m}}\right)$ and $q \equiv\left(\mathbf{q}, i \nu_{\mathrm{n}}\right)$ for convenience, the matrix for the selfenergy $\Sigma_{l l^{\prime}}(k)$ reads

$$
\Sigma_{l l^{\prime}}(k)=\frac{1}{\beta N} \sum_{k^{\prime}} V_{l l^{\prime}}\left(k-k^{\prime}\right) G_{l l^{\prime}}\left(k^{\prime}\right),
$$

where $\beta=\frac{1}{k_{B} T}$ is the inverse temperature, and the effective interaction $V_{l l^{\prime}}$ results from an infinite series over spin- and charge-fluctuations and is given by

$$
\begin{aligned}
V(q)= & \frac{3 U^{2}}{2}(1-U \chi(q))^{-1} \chi(q) \\
& +\frac{U^{2}}{2}(1+U \chi(q))^{-1} \chi(q) \\
& -U^{2} \chi(q) .
\end{aligned}
$$

Note, that $V$ and $\chi$ are $(2 \times 2)$ matrices, i.e. matrix inversion and multiplications have to be used. The bare particle-hole bubble $\chi_{l l^{\prime}}(q)$ consists of dressed Green's functions

$$
\chi_{l l^{\prime}}(q)=-\frac{1}{\beta N} \sum_{k} G_{l l^{\prime}}(k+q) G_{l^{\prime} l}(k) .
$$

In Eq. 8, the Green's functions are determined selfconsistently by solving the set of coupled equations Eqs. (5, 6.,7,8). During the self-consistency cycle, we fix the onsite energy $\delta$ and the particle number $n_{1}=1-x_{1}$ of the first layer, while the chemical potential $\mu$ and the particle number of the second plane $n_{2}=1-x_{2}$ are determined at each step. It turns out that the total particle number $n=n_{1}+n_{2}$ does not essentially depend on temperature, which makes the physical interpretation of our numerical results more straightforward. To avoid the uncertainties related to a numerical analytical continuation of correlation functions from the imaginary Matsubara to real frequencies, we use the recently proposed real-frequency approach to the FLEX approximation.22

In the following, we shall mainly focus our attention on two different parameter sets in order to mimic a situation with strong and weak antiferromagnetic fluctuations, respectively. Specifically, we use a parameter set, for simplicity labeled by "A", with $t^{\prime}=-0.38 t, t^{\prime \prime}=-0.06 t$ and a second one, labeled by "B", with $t^{\prime}=-0.20 t, t^{\prime}=0.15 t$. As will be shown below, the parameter set A corresponds to a system with pronounced antiferromagnetic fluctuations at low temperature, while B has much weaker ones.

\section{RESULTS}

In order to investigate the effects of different charge carrier concentrations in coupled bilayer systems, we start the discussion with the $\delta$ dependence of the doping of the second plane, $x_{2}(\delta)$. Here, the doping of the first plane $x_{1}$ and the on-site energy $\delta$ are independent variables for us, while $x_{2}$ comes out from the self-consistent calculation. In Fig. 11 we present $x_{2}(\delta)$ for both parameter sets $\mathrm{A}$ and $\mathrm{B}$ for different doping levels of the first plane as a function of $\delta$. From Fig. 1 it can be seen that the hole doping of the second plane is directly proportional to its on-site energy $\delta$. Note that the constant of proportionality, i.e. the slope $\partial x_{2} / \partial \delta$, is almost independent on $t^{\prime}, t^{\prime \prime}$ and $x_{1}$, because the particle number difference $\delta n=n_{1}-n_{2}$ between both planes is mainly governed by the energy dependence of the effective "chemical potentials" $\mu$ and $\mu-\delta$. On the other hand, correlation effects do play a role here, since the slope depends on $U / t$. Note that this energy difference is determined selfconsistently in our theory and therefore does not only 
depend on $\delta$, but varies, due to self-energy renormalizations, also with the strength of the Coulomb interaction $U$. The reason why we follow the strategy of keeping the doping of the first plane fixed and only change the doping of the second plane is that we want to investigate a possible induced coupling effect between the planes. The question is whether single-particle and magnetic properties of the first plane are influenced by the doping of the second plane.

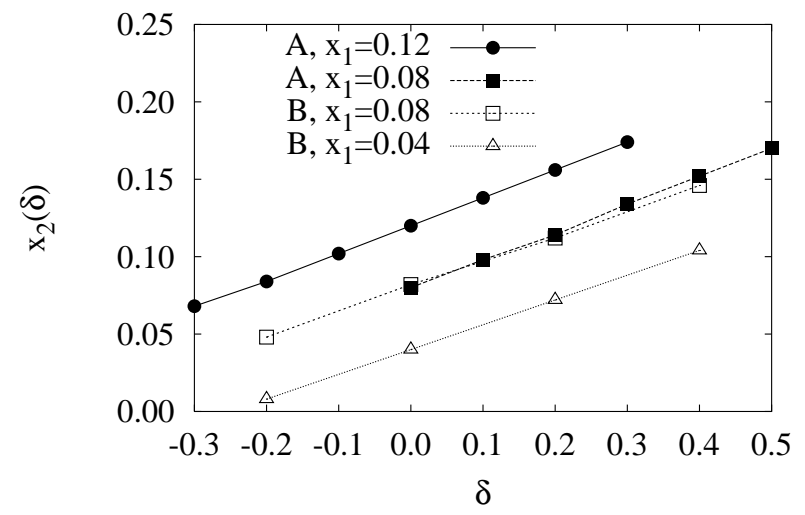

FIG. 1. Hole density of the second layer as a function of its on-site energy $\delta$ for parameter sets A and B (described in the text) and different dopings of the first layer $(T=0.02 t)$.

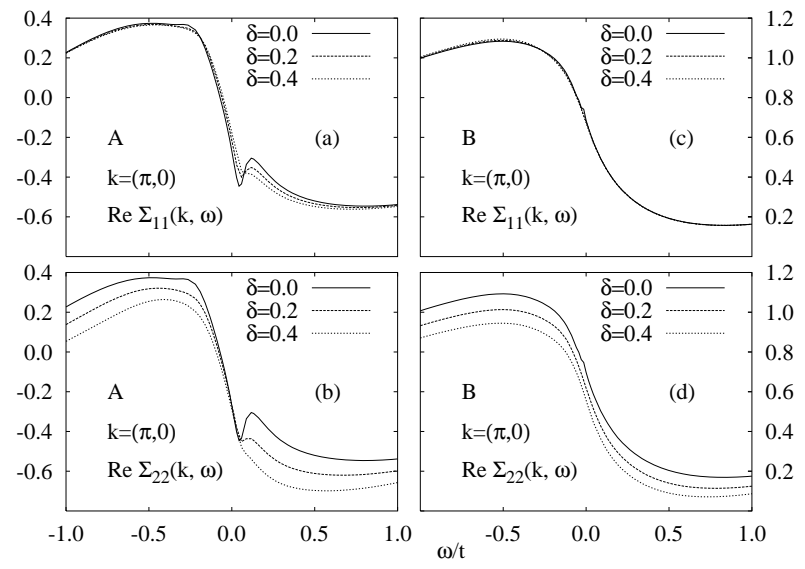

FIG. 2. Real part of the diagonal elements of the self-energy, $\operatorname{Re} \Sigma_{l l}(\mathbf{k}, \omega)$ for $\mathbf{k}=(\pi, 0)$ and $x_{1}=0.08, T=0.02 t$. (a) and (b) correspond to the parameter set A and (c) and (d) to the set B, respectively.

In Fig. 2, we show the real part of the diagonal elements of the self-energy $\Sigma_{l, l}(\mathbf{k}, \omega)$ with $\mathbf{k}=(\pi, 0)$ for both layers and both parameter sets A and B. The different curves in each panel are for various on-site energies $\delta$. Fig. 2(a) displays $\operatorname{Re} \Sigma$ for the first (less doped) layer with parameter set $\mathrm{A}$, i.e. for $t^{\prime}=-0.38 t, t^{\prime \prime}=0.06 t$. This figure shows that $\operatorname{Re} \Sigma_{11}$ indeed depends on $\delta$ although the hole concentration is not changed by $\delta$ in the first layer. This demonstrates that feedback effects due to the interlayer coupling modify also the properties of the plane where the charge carrier concentration is kept constant. The dip-like structure for small $\delta$ is a precursor of new quasiparticle states on the shadow of the Fermi surface due to strong antiferromagnetic fluctuations. This effect, which is strongest for the case of equivalent planes $(\delta=0)$, has been discussed in Refs. 17,18. An increase of $\delta$ and hence of the total hole doping of the system leads to a decrease of the interplanar antiferromagnetic coupling and thus of the dip structure. Panel (b) in this figure shows $\operatorname{Re} \Sigma_{22}((\pi, 0), \omega)$ for the second (heavily doped) layer. Since it is this plane which is primary altered by $\delta$ the influence of changing the on-site energy $\delta$ is, as expected, considerably more pronounced. These results show that the two layers are strongly connected and a change in carrier concentration of the second layer strongly influences the single-particle properties of the first layer as well, although the doping is unchanged here. However, whether the two planes are connected or not depends on the values of the parameters of the model. For example, a completely different situation is found for the parameter set labeled by B. For this choice, a variation of $\delta$ influences the second layer [panel (d) of Fig. 2], but has no effects on the self-energy of the quasi-particles in the first layer [panel (c)], indicating independent planes.

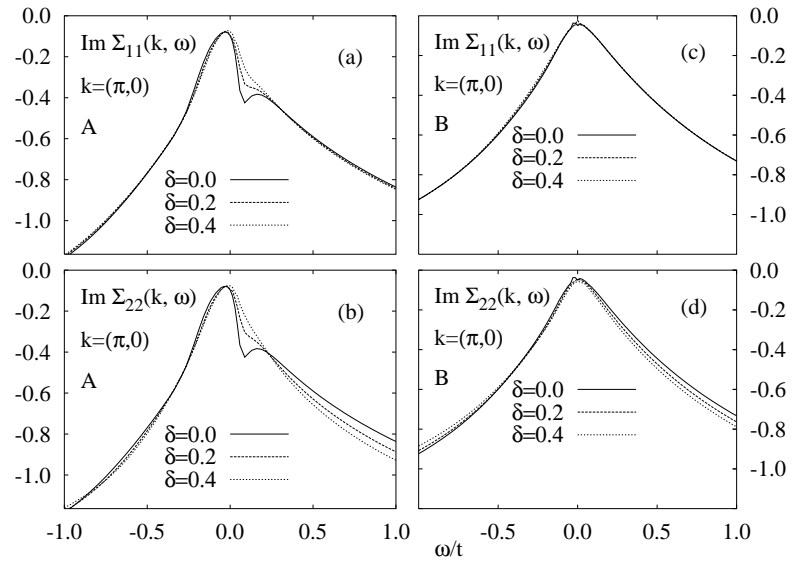

FIG. 3. Imaginary part of the diagonal elements of the self-energy, $\operatorname{Im} \Sigma_{l l}(\mathbf{k}, \omega)$ for $\mathbf{k}=(\pi, 0)$ and $x_{1}=0.08, T=0.02 t$

The connection between the two planes is also visible in the scattering rates which are related to the imaginary part of the self-energy and presented for both layers and both parameter sets in Fig. 3. For the imaginary part of the self-energy, which is more sensitive to low energy excitations, we observe an even closer connection between the two planes for parameter set A than for the real parts. In addition, these figures demonstrate that precursors of a spin density wave state around $(\pi, 0)$, for low $\delta$, are rather incoherent due to the strong scattering rates at these energies. Note also, that even the quasiparticles at the chemical potential $(\omega=0)$ suffer strong scattering, as indicated by the rather large values $\operatorname{Im} \Sigma_{l l}((\pi, 0), \omega=0)$. Similarly to the real part, the changes in the scatter- 
ing rates caused by $\delta$ are rather moderate for parameter set $\mathrm{B}$, even in the second layer which is directly altered by $\delta$ through its doping. Thus, both the real and the imaginary parts of the self-energy suggest a tendency of equalization between the layers for parameter set $\mathrm{A}$, but not for B.

Nevertheless, the two planes turn out to be disconnected for regions far from the Fermi surface, e.g. close to $\mathbf{k}=(0,0)$, even for data set $\mathrm{A}$.

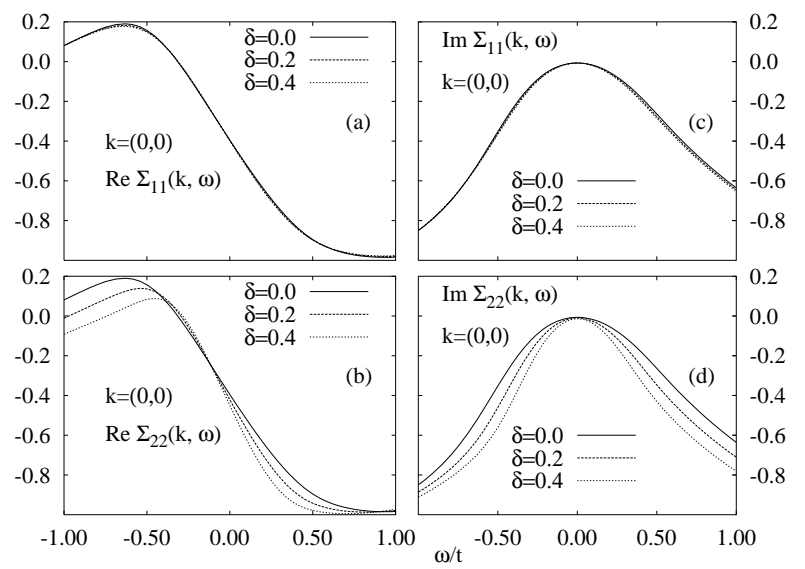

FIG. 4. Real and imaginary part of the two diagonal elements of the self-energy for parameter set $\mathrm{A}$ and for $\mathbf{k}=(0,0)$.

The real and imaginary parts of the self-energy for parameter set $\mathrm{A}$ and $\mathbf{k}=(0,0)$ are presented in Fig. A. As can be seen in part (a) and (c) of this figure, changes in the on-site energy $\delta$ of the second plane have almost no effect on the first plane. The two planes are thus connected only for momenta close to the Fermi surface which are strongly affected by antiferromagnetic fluctuations at low energy and in particular for hot quasiparticles states around $(\pi, 0)$.

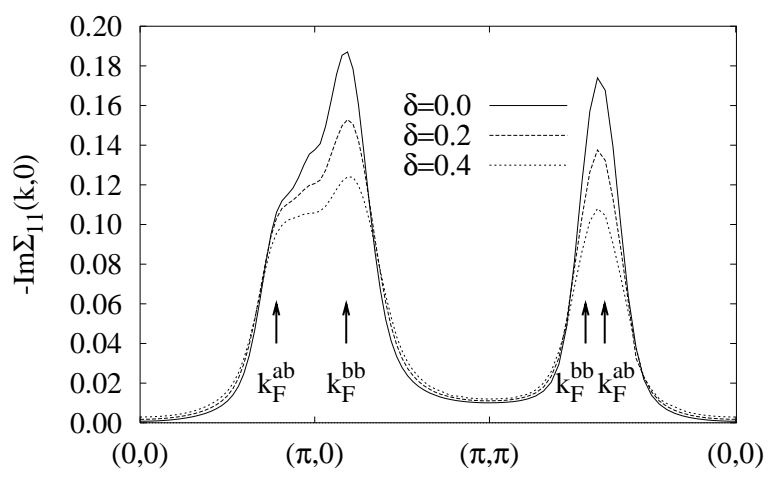

FIG. 5. Scattering rate in the first plane $-\operatorname{Im} \Sigma_{11}(\mathbf{k}, \omega=0)$ along the standard path in the Brillouin zone for parameter set A with $x_{1}=0.08$, $T=0.02 t, \delta / t=0.0,0.2,0.4$. The arrows indicate crossing of the anti-bonding $\left(\mathbf{k}_{F}^{a b}\right)$ and bonding $\left(\mathbf{k}_{F}^{b b}\right)$ Fermi surfaces (see also Fig. 10).

To further elucidate the momentum-resolved equaliza- tion effects for parameter set A, we show in Fig. 5 the scattering rates at the Fermi energy for the first plane, namely $-\operatorname{Im} \Sigma_{11}(\mathbf{k}, \omega=0)$ along the standard path in the Brillouin zone, for different values of the on-site energy $\delta$ of the second plane. This figure demonstrates that the scattering rates are strongly modified at the Fermi surface with strong effects in the regions close to $(\pi, 0)$. On these regions, the large number of states associated with the flat bands produces strong scattering processes, whenever the interaction connects the van Hove regions of the bonding and antibondig band. We stress again that the variation of the first plane is solely caused by its correlation with the second plane since the doping of the first plane is kept constant. The corresponding real part of the self-energy (not shown) also reveals coupling effects for all $\mathbf{k}$ close to $\mathbf{k}_{F}$.

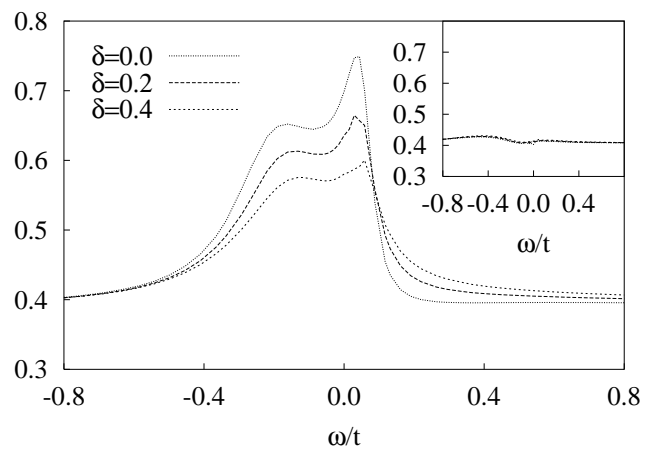

FIG. 6. Effective interplane hopping $t_{\perp}^{\text {eff }}(\mathbf{k}, \omega)$ as a function of the onsite energy $\delta$ for $t_{\perp}=0.4 t, x_{1}=0.08, T=0.02 t$ for parameter set $A$. The inset shows the results for parameter set $\mathrm{B}$ on the same scale.

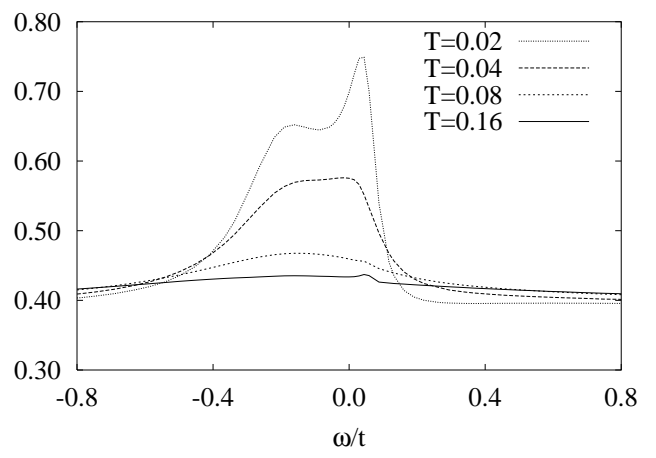

FIG. 7. Effective interplane hopping $t_{\perp}^{\text {eff }}(\mathbf{k}, \omega)$ as a function of temperature for parameter set A with $x_{1}=0.08$, $\delta=0.0$. Note the stronger dependence on $T$ than on $\delta$ when compared to Fig. 6 .

We now address the question of how much the hopping $t_{\perp}$ between the planes is affected by the interaction. Dyson's equation (Eq. 5) suggests a momentum- and 
energy-dependent effective interlayer hopping according to

$$
t_{\perp}^{\mathrm{eff}}(\mathbf{k}, \omega)=t_{\perp}+\operatorname{Re} \Sigma_{12}(\mathbf{k}, \omega)
$$

Like the real and imaginary parts of the diagonal elements of $\Sigma(\mathbf{k}, \omega)$, this quantity is strongly affected for $\mathbf{k} \approx(\pi, 0)$, therefore, we restrict ourselves to this momentum.

In Fig. 6 we show $t_{\perp}^{\text {eff }}(\mathbf{k}, \omega)$ for parameter set $A$ at a fixed temperature $T=0.02 t$ for a series of on-site energies $\delta$, while the inset presents the same quantities for parameter set B. Looking at Fig. 6, we observe that $t_{\perp}$ is strongly renormalized close to the chemical potential $(\omega=0)$, and even more important, that the antiferromagnetic fluctuations again enhance the interlayer connection, in this case represented by the effective hopping $t^{\text {eff }}$. On the contrary, $t_{\perp}$ is essentially unrenormalized, independently of the value of $\delta$, in the presence of the weak antiferromagnetic fluctuations in parameter set $B$ (see inset of the figure). This result seems to stress once more the fact that the planes are strongly connected for the "antiferromagnetic" parameter set A, whereas they are essentially independent for the parameter set B. We thus focus our attention on parameter set $\mathrm{A}$ where the effects are stronger. For $\delta=0$ the effective hopping $t_{\perp}^{\mathrm{eff}}((\pi, 0), 0) \approx 0.8 t$ is roughly twice as large as the bare $t_{\perp}=0.4 t$. Thus, the hopping between the planes is amplified rather than blocked by electronic correlations.

Fig. 6 also shows that the renormalization of $t_{\perp}$ decreases with increasing on-site energy $\delta$, which causes the second plane to be less magnetic. However, an increase of $\delta$ has a surprisingly weak effect on $t_{\perp}^{\text {eff }}((\pi, 0), \omega)$ when compared with an increase of the temperature $T$, which is shown in Fig. 7. This strong temperature dependence of $t_{\perp}^{\text {eff }}((\pi, 0), \omega)$ shown in the figure suggests that the interlayer coupling is related to a small energy scale $\omega^{\star}$. For high temperatures, the thermal fluctuations destroy the correlations on the small energy scale $\omega^{\star}$.

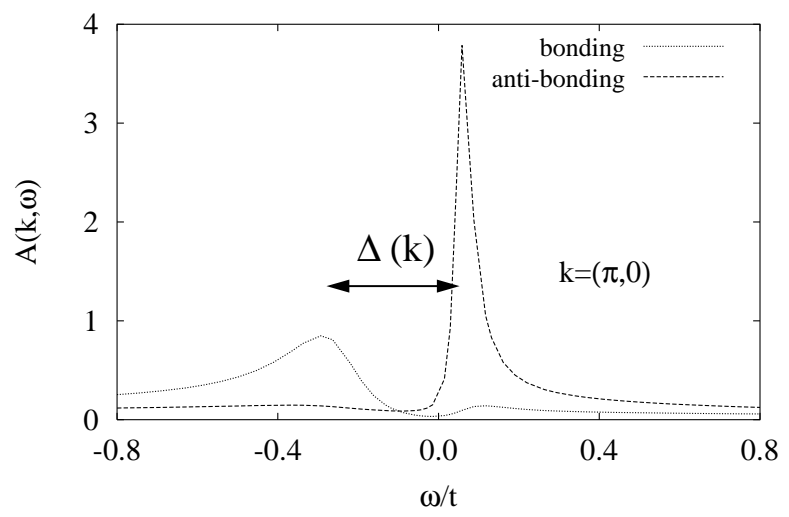

FIG. 8. Spectral functions $A(\mathbf{k}, \omega)$ of the bonding and anti-bonding bands at $\mathbf{k}=(\pi, 0)$ for parameter set $\mathrm{A}$ with $x_{1}=0.08, \delta=0, T=0.02 t$
Although the above discussion suggests an enhancement of $t_{\perp}$ due to interaction effects, the band splitting between the bonding and the anti-bonding band $\Delta(\mathbf{k})=\omega_{\mathbf{k}}^{+}-\omega_{\mathbf{k}}^{-}$goes in the opposite direction and is reduced with respect to its bare value $\Delta_{\mathrm{o}}=2 t_{\perp}$, in agreement with previous conjectures 23 25. Fig. 8 shows the spectral functions $A(\mathbf{k}, \omega)$ of the bonding and antibonding band for $\mathbf{k}=(\pi, 0)$ and indicates a renormalized band splitting $\Delta(\pi, 0) \approx 0.4 t=0.5 \Delta_{\mathrm{o}}$. Thus, while the interlayer hopping seems to be enhanced by about a factor of 2 at low energies in the presence of strong antiferromagnetic fluctuations, the band splitting behaves in the opposite way and it is reduced by about the same factor for this parameter set. This different behavior between $t_{\perp}^{\text {eff }}$ and $\Delta(\mathbf{k})$ is rather surprising, although it may be understood by the following argument. On the one hand, the quasiparticle interplane hopping without residual interaction, related to the off-diagonal energy term $t_{\perp}^{\mathrm{eff}}$, is enhanced due to the fact that quasiparticle of two neighboring sites on the two planes are nearly antiferromagnetically ordered and thus have a larger amplitude to hop. On the other hand, the whole hopping amplitude, related to $\Delta(\mathbf{k})$, is suppressed (by a larger factor than the enhancement of $\left.t_{\perp}^{\text {eff }}\right)$, due to the Hubbard repulsion $U$.

Equalization effects between the planes are also observed in two-particle quantities like the spin response as deduced from the spin-spin correlation function. This is given (in the layer representation) by

$$
\chi^{z z}(\mathbf{q}, \omega)=2[1-U \chi(\mathbf{q}, \omega)]^{-1} \chi(\mathbf{q}, \omega) .
$$

The static spin-spin correlation function $\chi_{l l}^{z z}(\mathbf{q}, \omega=0)$ along the standard path $(0,0) \rightarrow(\pi, 0) \rightarrow(\pi, \pi) \rightarrow(0,0)$ in the Brillouin zone is shown in Fig. 9 for $l=1$ and for $l=2$ for parameter set $\mathrm{A}$ and $\mathrm{B}$.
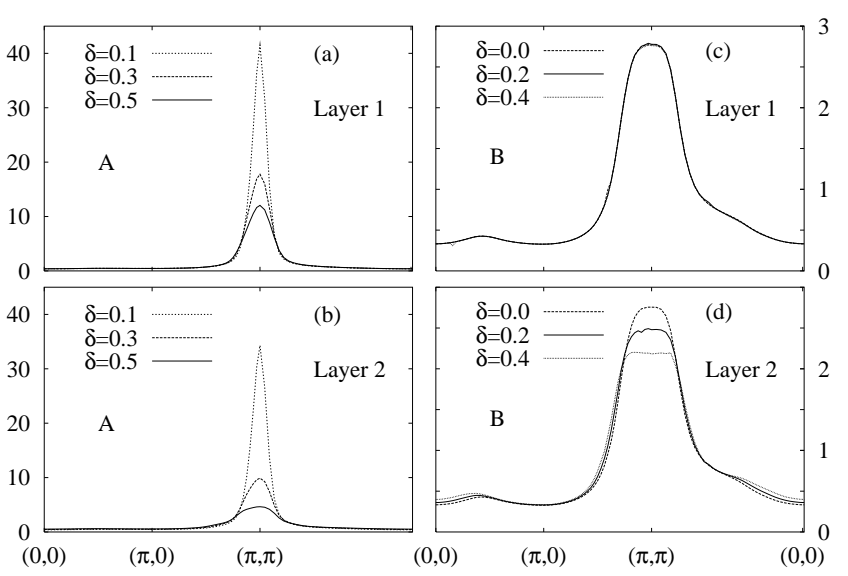

FIG. 9. Static spin susceptibility $\chi_{l l}^{z z}(\mathbf{q}, \omega=0)$ along the standard path in the Brillouin zone for the two layers and both parameter sets A and B. $\left(T=0.02 t, x_{1}=0.08\right)$

In the case of parameter set $\mathrm{A}$, the spin response in both planes is strongly peaked at $\mathbf{q}=(\pi, \pi)$ indicating 
considerable antiferromagnetism in the Hubbard planes. However, even more important is the strong dependence of $\chi_{11}^{z z}(\mathbf{q}, 0)$ on $\delta$ which is solely due to the interplane coupling since the doping in the first plane is fixed. In agreement with the effects observable in the single-particle spectrum represented by the self-energy, this clearly reveals a strong connection between the planes. Fig. 9(a) and (b) also show that the spin response is very sensitive to a variation of $\delta$ and the antiferromagnetism is suppressed if $\delta$ is increased. This is due to the increasing hole concentration in the total system which tears it away from half-filling where antiferromagnetism is strongest. The static spin response for the data set labeled by B is shown in Fig. 9(c) and (d). For this choice of parameters, the first plane is again completely disconnected from the second one since a change of $\delta$ only influences the spin response of the second plane while that of the first plane is not affected at all. A clue towards the understanding of the difference between the parameter sets $\mathrm{A}$ and $\mathrm{B}$ is already found in the behavior of the spin response. Comparing Fig. 9 (a)-(b) with Fig. 9 (c)-(d) reveals a considerable (one order of magnitude) smaller value for the static spin response at $(\pi, \pi)$ in the second case. Furthermore, the spin correlation length $\xi$, which is the inverse of the half width at half maximum of $\chi^{z z}(\mathbf{q}, 0)$ is much smaller (of the order of 1 lattice spacing) for parameter set B. This implies, as expected, that the interplane connection is intimately related to strong antiferromagnetic correlations in the planes.
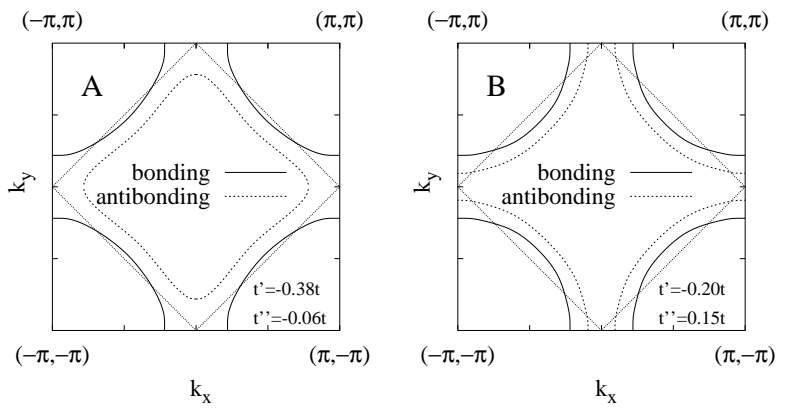

FIG. 10. Bonding and anti-bonding Fermi surfaces for parameter sets A (left panel) and B (right panel) with $x_{1}=0.08, \delta=0.2, T=0.02 t$.

The strength of the antiferromagnetic fluctuations are in turn quite sensitive to the shape of the Fermi surface, especially if large regions of the Fermi surface can be linked by the antiferromagnetic momentum $\mathbf{Q}=(\pi, \pi)$ 26 . Keeping this in mind, the differences between parameter set A and B are caused by their different Fermi surfaces. These are shown in Fig. 10. While the antibonding and bonding Fermi surfaces associated with parameter set A are closed around $(0,0)$ and $(\pi, \pi)$, respectively, both Fermi surfaces of B are closed around $(\pi, \pi)$. Even more important is the fact that large regions of both Fermi surfaces in case A may be connected by $\mathbf{Q}$. These regions are close to $(\pi, 0)$, i.e. close to the van Hove singularities in the density of states, thus opening various channels for antiferromagnetic scattering processes.

\section{RELATION TO EXPERIMENTS}

We now turn to the question how our theoretical calculations compare with experimental results. We thus concentrate our attention to NMR experiments on $\mathrm{Y}_{2} \mathrm{Ba}_{4} \mathrm{Cu}_{7} \mathrm{O}_{15}$ performed by Stern et al. experimentally relevant quantities are the spin-lattice relaxation time $T_{1}$ and the Gaussian contribution $T_{2 G}$ to the nuclear spin-spin relaxation time $T_{2}$.

As pointed out by Shastry27 and Mila and Rice 28, the spin-lattice relaxation time $T_{1}$ is related to the spin susceptibility $\chi^{z z}$, via the expression:

$$
\frac{1}{T_{1} T}=\lim _{\omega \rightarrow 0} \frac{1}{2 N} \frac{k_{B}}{\hbar} \sum_{\mathbf{q}} F_{c}(\mathbf{q}) \frac{\operatorname{Im} \chi^{z z}(\mathbf{q}, \omega)}{\hbar \omega},
$$

where $F_{c}(\mathbf{q})$ is the form factor resulting from the Fourier transform of the hyperfine interaction

$$
F_{c}(\mathbf{q})=\left\{A_{a b}+2 B\left[\cos q_{x}+\cos q_{y}\right]\right\}^{2} .
$$

Thus, $T_{1}$ probes the slope of the imaginary part of $\chi^{z z}(\mathbf{q}, \omega)$ for $\omega \rightarrow 0$. In contrast to this, the Gaussian component of the transverse relaxation time $T_{2 G}$ depends on the static susceptibility and is given by

$$
\begin{aligned}
T_{2 G}^{-2}= & \frac{0.69}{128 \hbar^{2}}\left[\frac{1}{N} \sum_{\mathbf{q}} F_{\text {eff }}^{2}(\mathbf{q}) \chi^{z z 2}(\mathbf{q}, 0)\right. \\
& \left.-\left(\frac{1}{N} \sum_{\mathbf{q}} F_{\text {eff }}(\mathbf{q}) \chi^{z z}(\mathbf{q}, 0)\right)^{2}\right]
\end{aligned}
$$

as pointer-out independently by Takigawa 29 and Thelen and Pine 30 . While Eq. 13 applies for the diagonal elements of $\chi_{l l}^{z z}$, i.e. for the in-plane relaxation rates, the corresponding inter-layer relaxation rate $1 / T_{2 G}^{12}$ needs not to be corrected by the self-interacting hyperfine interaction and thus reads:

$$
\left[T_{2 G}^{12}\right]^{-2}=\frac{0.69}{128 \hbar^{2}} \frac{1}{N} \sum_{\mathbf{q}} F_{\text {eff }}^{2}(\mathbf{q})\left[\chi_{12}^{z z}(\mathbf{q}, 0)\right]^{2}
$$

The form factor $F_{\text {eff }}(\mathbf{q})$ in the last two equations is simply obtained form Eq. (12) by replacing $A_{a b}$ with $A_{c}$. Although we are studying inequivalent Hubbard planes, we assume for simplicity that the hyperfine constants, which are usually extracted from Knight shift experiments, are identical in both planes. This is supported by the rather moderate variations of the hyperfine coupling constants for different cuprate superconductors. Quantitative differences may be obtained if one takes into account different hyperfine constant, although the temperature dependence should not change. To be specific, we adopt here the values recently given in an analysis of NMR exper iments on YBCO and LSCO by Barzykin and Pines 31 
and set $A_{a b}=0.84 B, A_{c}=-4 B$ and the energy scale $B=3.82 \times 10^{-7} \mathrm{eV}$. Note, that since NMR probes the local environment of the spins, all momenta $\mathbf{q}$ contribute to the relaxation times although the main contributions come from the regions $\mathbf{q} \cong(\pi, \pi)$. Hence, the behavior of the NMR relaxation times are strongly influenced by the antiferromagnetic response of the system.

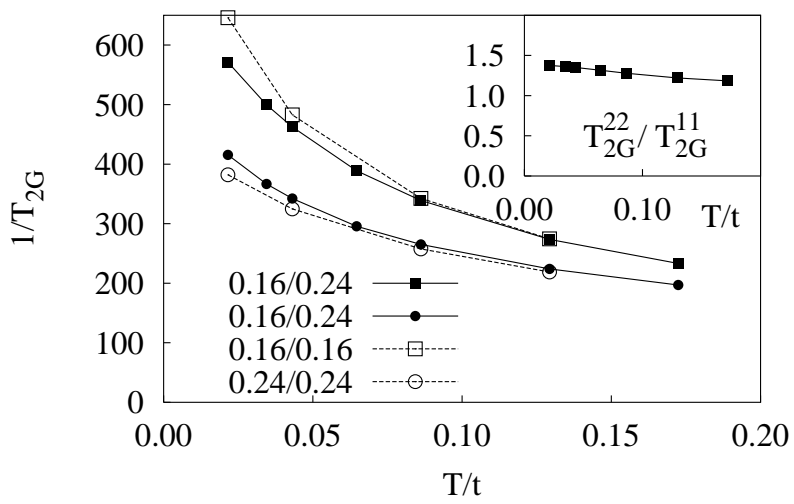

FIG. 11. Temperature dependence of the Gaussian component of the spin-spin relaxation rate $T_{2 G}$ of a bilayer system consisting of two different layers with doping $x_{1}=0.16$ and $x_{2}=0.24$, respectively $(\delta=0.4)$. For comparison, we show also the result for two bilayer systems with equivalent layers with doping $x_{1}=x_{2}=0.16$ and $x_{1}=x_{2}=0.24$, respectively. The other parameters for all curves are: $t^{\prime}=-0.38 t, t^{\prime \prime}=-0.06 t$. The inset shows the ratio $T_{2 G}^{22} / T_{2 G}^{11}$ for the case of inequivalent layers.

We start the discussion with the transverse relaxation time $T_{2 G}$. In Fig. 11 we show $1 / T_{2 G}$ as a function of temperature for both planes of the system with inequivalent layers in comparison with the corresponding data for two corresponding bilayer systems with equivalent layers, one with the same doping as the first layers and one with the same doping as the second layer of the first system. These results for the different layers are obtained with Eq. 13 by substituting $\chi^{z z}$ with element (11) or (22) from Eq. 10. The filled (open) symbols in this figure are related to the system with inequivalent (equivalent) layers. Furthermore, the squares represent the data for planes with a hole doping of $x=0.16$ and the bullets planes with doping $x=0.24$. From Eq. 13 it is seen that a system with rather strong magnetism and hence large values of $\chi^{z z}(\mathbf{q})$ exhibits large relaxation rates $1 / T_{2 G}$. This explains the differences between the layers with $x=0.16$ and $x=0.24$, i.e. with different doping levels, whereby the heavily doped plane shows a smaller relaxation rate. The most striking result is that data for a plane with a given doping also depend on whether that plane is coupled with an equivalent one or with a more or less doped one. The heavily doped plane $\left(x_{2}=0.24\right)$ of the system with inequivalent layers shows stronger magnetic fluctuations than the plane in the corresponding system with equivalent bilayers $\left(x_{1}=x_{2}=0.24\right)$. Similarly, the magnetism of the lower doped plane $\left(x_{1}=0.16\right)$ is reduced with respect to the corresponding equivalent-layer system $\left(x_{1}=x_{2}=0.16\right)$ due to the coupling to a stronger doped plane. Thus, the magnetic fluctuations of the two inequivalent planes with different carrier concentration tend to be equalized by interplane coupling effects. A related effect has been detected also in Quantum-MonteCarlo simulations of coupled Hubbard planes carried out by Scalettar et al.19, in which the two planes have different chemical potentials and one plane is adjusted to half filling. In this case, the antiferromagnetic susceptibility of the half-filled plane is reduced by the coupling with the doped plane. The authors explain this by the fact that processes in which holes hop from the doped layer into the half filled one are energetically more favorable than virtual hopping processes associated with the magnetic exchange $J \propto t^{2} / U$.
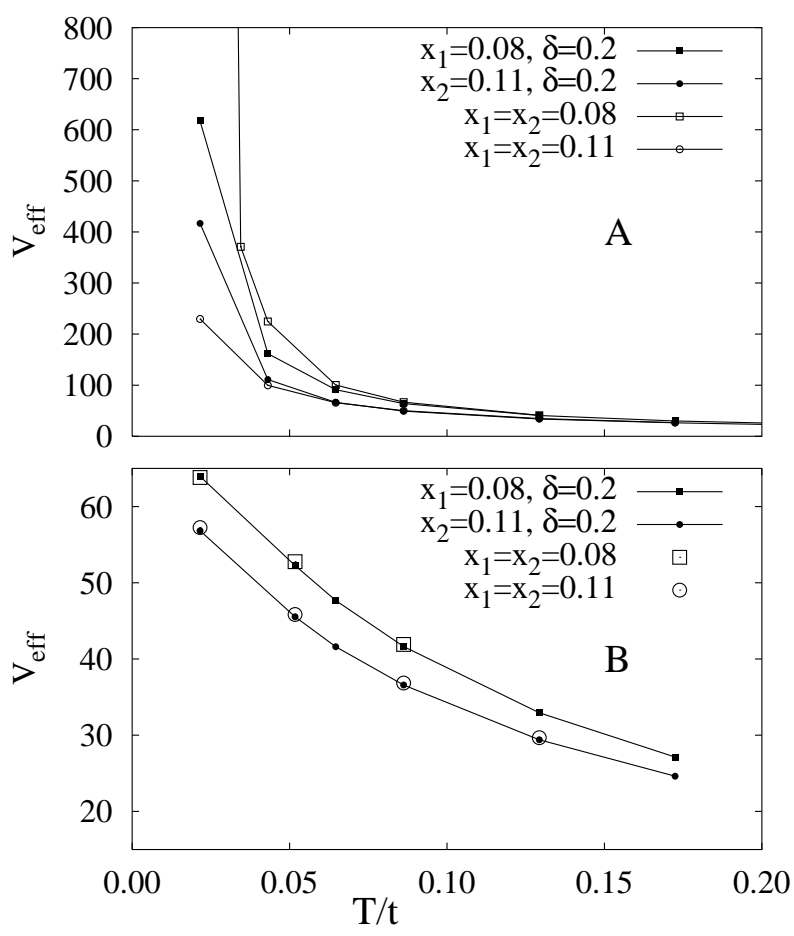

FIG. 12. Effective interaction $V_{\text {eff }}(\mathbf{Q}, \omega=0)$ for a system with inequivalent layers $\left(x_{1}=0.08\right.$ and $\left.x_{2}=0.11\right)$ in comparison with the corresponding bilayer systems with $x_{1}=x_{2}=0.08$ and $x_{1}=x_{2}=0.11$. The upper panel shows the results for parameter set $\mathrm{A}$ and the lower for $\mathrm{B}$.

The effects discussed above are also visible in other quantities, like the effective interaction $V_{\text {eff }}(\mathbf{q}, \omega)$, which is proportional to the spin susceptibility in our approximation. For this quantity, we can see that the stronger the antiferromagnetic fluctuations are in the planes, the stronger is the tendency to equalization of the two planes. In Fig. 12(a) we show $V_{\text {eff }}(\mathbf{q}=(\pi, \pi), \omega=0)$ as a func- 
tion of temperature for the parameter set A as measured in the two layers of the system with inequivalent layers. In the same figure, we also report for comparison the data for the two corresponding systems with equivalent layers with their doping adjusted to the one of each of the two layers of the first system. In Fig. 12(b) we show the same comparison for the systems with parameter set B. The difference is striking. For parameter set $\mathrm{A}, V_{\text {eff }}$ of each of the two layers in the system with inequivalent layers is considerably different from $V_{\text {eff }}$ in the corresponding system with equivalent layers and tend to be equalized for the two layers. On the other hand, for the less antiferromagnetic parameter set $\mathrm{B}, V_{\text {eff }}$ calculated on a given layer of the system with inequivalent layers is essentially the same as the one calculated on the system with the same doping and equivalent layers.

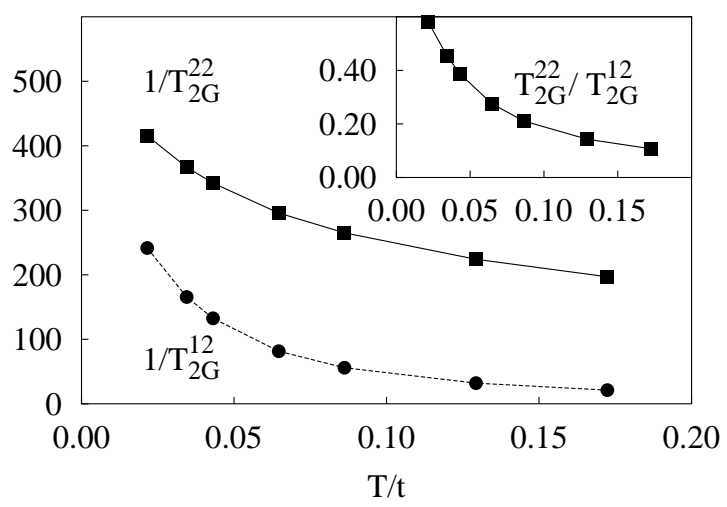

FIG. 13. Intra- and inter-plane spin-spin relaxation rates $1 / T_{2 G}^{22}$ and $1 / T_{2 G}^{12}$, respectively. The inset shows the ratio between the two relaxation rates $\left(x_{1}=0.16, x_{2}=0.24, \delta=0.4\right)$.

Qualitatively, the experimental situation for the system with inequivalent layers, $\mathrm{Y}_{2} \mathrm{Ba}_{4} \mathrm{Cu}_{7} \mathrm{O}_{15}$, appears to be described by parameter set A. Indeed, NMR experiments by Stern et al. 3 on $1 / T_{2 G}$ for the two layers of $\mathrm{Y}_{2} \mathrm{Ba}_{4} \mathrm{Cu}_{7} \mathrm{O}_{15}$ and for the two associated systems with equivalent layers, $\mathrm{YBa}_{2} \mathrm{Cu}_{3} \mathrm{O}_{7}$ and $\mathrm{YBa}_{2} \mathrm{Cu}_{4} \mathrm{O}_{8}$, show the same behavior as observed in Fig. 11 and in Fig. Fig. 12(a), if one identifies the behavior of $1 / T_{2 G}$ with the one of $V_{\text {eff }}$. Both experimental and theoretical results show a strong increase of $1 / T_{2}$ as the temperature is lowered. However, we do not obtain the decrease of $1 / T_{2 G}$ below $T_{s g} \approx 100 \mathrm{~K}$ which is attributed to the opening of a gap in the spin excitation spectrum, since this region is probably unaccessible by our approximation. Another experimental observation is that the spin-lattice relaxation rate $1 / T_{2 G}$ has the same temperature dependence in the two planes of $\mathrm{Y}_{2} \mathrm{Ba}_{4} \mathrm{Cu}_{7} \mathrm{O}_{15}$. This has been deduced from the ratio $R=\left(1 / T_{2 G}^{124}\right) /\left(1 / T_{2 G}^{123}\right)$ of $1 / T_{2 G}$ in the two planes, which turned out to be temperature independent and approximately $R \approx 1.4-1.5$. Since the 124 plane in the coupled layer structure of $\mathrm{Y}_{2} \mathrm{Ba}_{4} \mathrm{Cu}_{7} \mathrm{O}_{15}$ is the one with lower doping, the $\mathrm{CuO}_{2}$-layer from the 123 block corresponds to the second plane in our theoretical study. The calculated values for $R=T_{2 G}^{(22)} / T_{2 G}^{(11)}$ are presented in the inset of Fig. 11. It turns out that $R$ is almost independent of the temperature $T$, in agreement with the experimental finding.

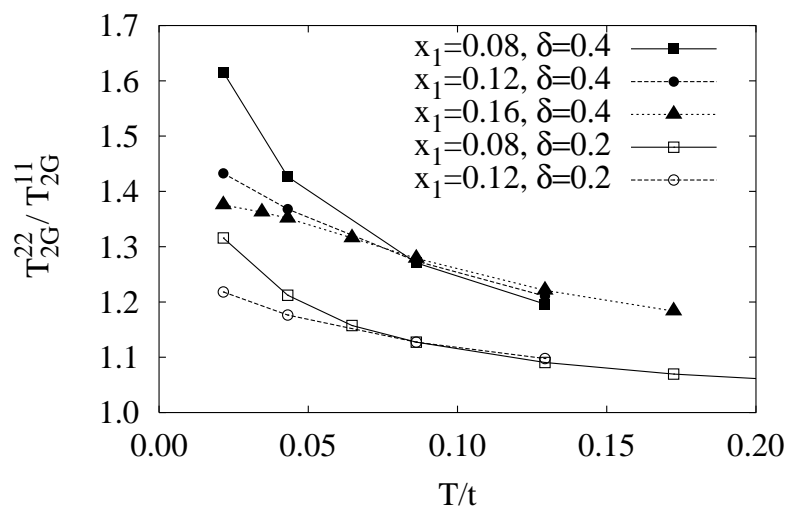

FIG. 14. The ratio $R(T)=T_{2 G}^{(22)} / T_{2 G}^{(11)}$ for parameter set A and various values of $x_{1}$ and $\delta$.

In fact, a systematic study shows that the ratio $R$ is mainly controlled by the on-site energy $\delta$ and thus by the difference of particle densities, $\delta n=n_{1}-n_{2}$, between the two planes. On the other hand, the temperature dependence of $R(T)$ is sensitively related to the doping of the first layer: for low doping $x_{1}, R(T)$ shows an upturn with decreasing $T$ while it is essentially constant for large values of $x_{1}$ (see Fig. 14). Thus, the experimental data for $R(T)$ imply a rather large doping $x_{1}$ of the first layer in connection with a large on-site energy difference $\delta$ or, equivalently, filling difference $\delta n$. On the other hand, the doping cannot be too large because otherwise no tendency to equalization would be observable. An optimal choice for the doping levels in the coupled system turns out to be $x_{1}=0.16$ and $x_{2}=0.24$, as shown in Fig. 11 .

For the same parameter choice, we compare the temperature dependence of the in-plane relaxation rate $1 / T_{2 G}^{22}$ with the inter-plane one $1 / T_{2 G}^{12}$, which is calculated using Eq. 14. The latter quantity has been measured using NQR_SEDOR experiments 4 , as suggested by Monien and Rice5. The apparent feature in these experiments is that the inter-plane relaxation rate increases faster for decreasing temperature than the in-plane one, as seen from the temperature dependence of the ratio $R_{\text {SEDOR }}(T)=T_{2 G}^{22} / T_{2 G}^{12}$. Our theoretical calculations displayed in Fig. 13 clearly reproduce the qualitative behavior observed experimentally. However, we do not observe the saturation effect for very low temperatures as seen in experiments and which is most probably due to the opening of the spin gap. 


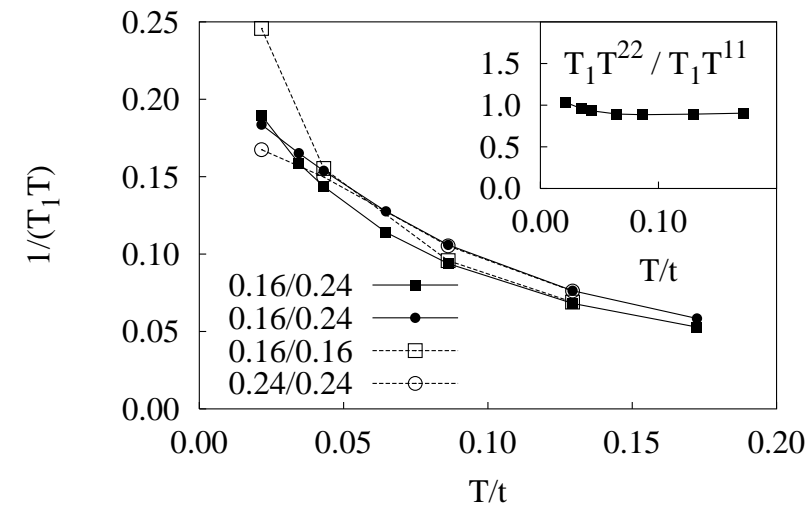

FIG. 15. Temperature dependence of the spin-lattice relaxation rate $1 /\left(T_{1} T\right)$ of a system with inequivalent layers compared to the corresponding systems with equivalent layers and the same doping concentration, the inset shows the ratio of $1 /\left(T_{1} T\right)$ for the two layers of the inequivalent-layer system (cf. Fig. 11).
A similar study of the spin-lattice relaxation time $T_{1}$ on $\mathrm{Cu}$-sites shows less clear coupling effects between the two layers, both theoretically and experimentally. The experimental results 2 show almost no difference between the $1 /\left(T_{1} T\right)$ data for the $123 / 124$ layers in $\mathrm{Y}_{2} \mathrm{Ba}_{4} \mathrm{Cu}_{7} \mathrm{O}_{15}$ and the corresponding layers in the $\mathrm{YBa}_{2} \mathrm{Cu}_{3} \mathrm{O}_{7}$ and $\mathrm{YBa}_{2} \mathrm{Cu}_{4} \mathrm{O}_{8}$ systems, respectively. Deviations can be seen only for rather low temperatures $T \lesssim T_{\text {sg }}$. Above $T_{s g}$, the ratio $R_{1}(T)=\left(T_{1} T^{123}\right) /\left(T_{1} T^{124}\right)$ deduced from the experiments is again essentially temperature independent and is about 1.4. In Fig. 15 we show the calculated results for $1 /\left(T_{1} T\right)$ obtained with Eq. 11 for the same parameters as in Fig. 11. Here, we observe that the coupling effects between the planes are not as strong as for the spin-spin relaxation rate $1 / T_{2 G}$. In fact, especially for the plane with the larger doping $(x=0.24)$ $1 /\left(T_{1} T\right)$ seems to deviate from the value of the system with equivalent layers only for rather small temperatures $T / t \approx 0.05$. On the other hand, the plane with lower doping $(x=0.16)$ shows deviations already for higher temperatures $T / t \approx 0.08$. Even more surprising is the result for the ratio $R_{1}(T)$, shown in Fig. 11. Here, the plane with the higher doping shows a larger spin-lattice relaxation rate $1 /\left(T_{1} T\right)$, in contradiction with the simple expectation that lower doping should result in stronger antiferromagnetic fluctuations. The expected value $R_{1}>1$ is restored only for low $T \lesssim 0.04 t$ in our calculation. The deviation from the experimental ratio $R_{1} \approx 1.4$ may be caused by the incorrect assumption that the hyperfine interaction constants are the same in both planes.
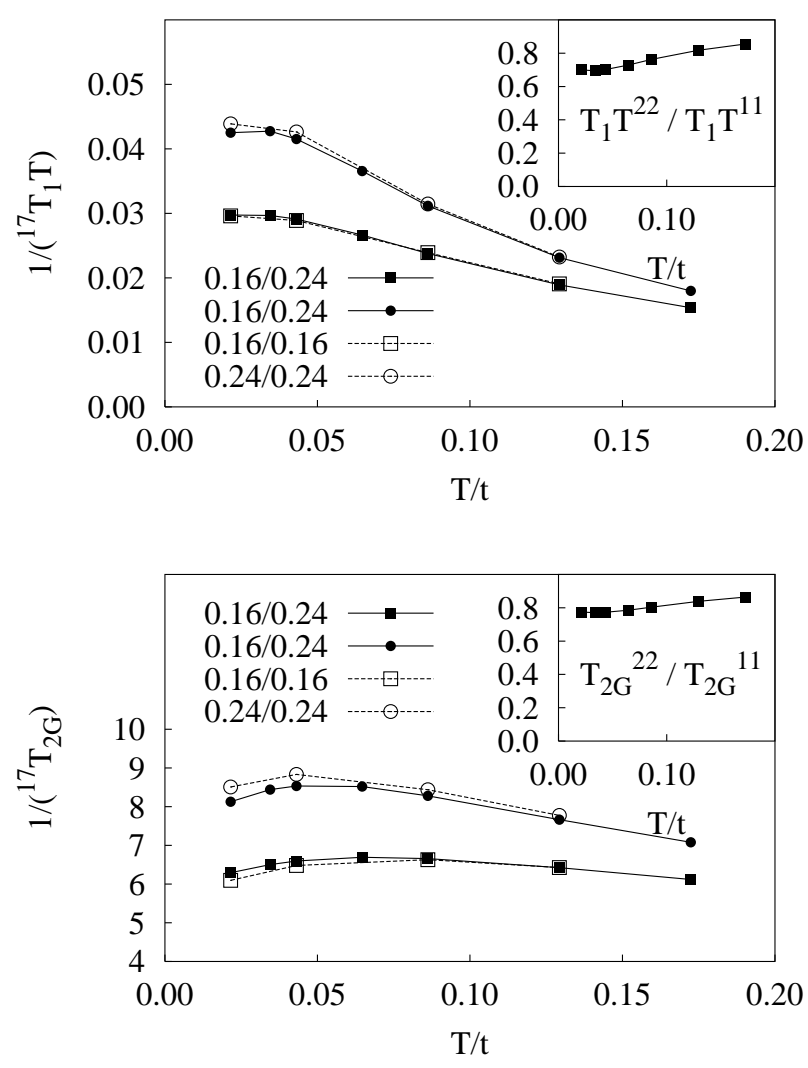

FIG. 16. Temperature dependence of the spin-lattice relaxation rates $1 /\left({ }^{17} T_{1} T\right)$ and of the spin-spin relaxation rates $1 /\left({ }^{17} T_{2 G}\right)$ on oxygen sites. Like in Fig. 11 and Fig. 15, the results for a system with inequivalent layers are compared with the corresponding systems with equivalent layers and the same doping (the parameters are the same as in Fig. 13).

Finally, we have calculated the relaxation times ${ }^{17} T_{1}$ and ${ }^{17} T_{2 G}$ on oxygen sites. Like the corresponding relaxation times on copper sites, these quantities are related to the slope for $\omega \rightarrow 0$ of the imaginary part of the spin susceptibility, and by its real part, respectively. The appropriate form factor which enters Eqs.11,13 and which is given by ${ }^{17} F(\mathbf{q})=2 C^{2}\left[1+0.5\left[\cos \left(q_{x}\right)+\cos \left(q_{y}\right)\right]\right]$ with two different constants for $T_{1} T$ and $T_{2 G}$, suppresses scattering events transfering momentum $\mathbf{q}=(\pi, \pi)$ related to magnetic fluctuations and instead favors those with $\mathbf{q}$ around $(0,0)$. The spin-lattice and spin-spin relaxation times on oxygen sites hence mainly probe the center of the Brillouin zone. We already know from the static spin susceptibility shown in Fig. 9 that the equalization effects are much weaker for $\mathbf{q} \cong(0,0)$ as compared with $(\pi, \pi)$. This weaker connection between the layers at $\mathbf{q} \cong(0,0)$ is equally pronounced for the dynamical susceptibility and thus leads to the oxygen relaxation rates $1 /\left({ }^{17} T_{1} T\right)$ and $1 /\left({ }^{17} T_{2 G}\right)$ shown in Fig. 16. Here, we find that the layers of the system consisting of two inequivalent layers behave almost like the corresponding planes in the systems with equivalent layers and the two inequivalent layers are essentially disconnected. We thus 
predict that, within a purely magnetic scenario, experimental measurements of the oxygen relaxation times in 123 and 124-layers of $\mathrm{Y}_{2} \mathrm{Ba}_{4} \mathrm{Cu}_{7} \mathrm{O}_{15}$ should behave, as a function of temperature, like those of the corresponding layers in $\mathrm{YBa}_{2} \mathrm{Cu}_{3} \mathrm{O}_{7}$ and $\mathrm{YBa}_{2} \mathrm{Cu}_{4} \mathrm{O}_{8}$, respectively. In other words, if one would detect a different behavior of $1 /\left({ }^{17} T_{1} T\right)$ in $\mathrm{Y}_{2} \mathrm{Ba}_{4} \mathrm{Cu}_{7} \mathrm{O}_{15}$ compared to the two parent compounds. it would be a strong indication for a nonmagnetic coupling of the two layers.

\section{CONCLUSIONS}

In summary, we have studied a microscopic model consisting of two inequivalent Hubbard planes which are connected by an interlayer hopping $t_{\perp}$. We have shown that magnetic and single-particle fluctuations of the two layers are connected and tend to be equalized if the antiferromagnetic fluctuations within the layers are strong. If the antiferromagnetic correlation length is less than 1-2 lattice spacings, which happens for high temperatures, large doping or bandstructure parameters with inefficient magnetic coupling between the two Fermi surface sheeds, we find that the two inequivalent layers are disconnected and keep their individual properties. However, once the antiferromagnetic correlations in the layer with smaller charge carrier concentration is sufficiently large, the single-particle excitations for momenta close to the Fermi surface and, in particular, around the hotspots as well as the magnetic excitations of the two layers are strongly connected. The whole system reacts, despite its inhomogenious charge density, magnetically as a single system. In this case, the interlayer antiferromagnetic susceptibility, measured by $T_{2 G}^{12}$, increases for decreasing temperatures more strongly than the individual inplane susceptibilities. These trends are in agreement with the experimenmtal observation by Stern et a demonstrating that it is sufficient, for an understanding of the magnetic interlayer coupling, to use a singleparticle interlayer hopping element. Furthermore, we expect from our analysis of the oxygen NMR relaxation rate that, in distinction to the Cu-relaxation rates, the magnetic connection between the two layers will barely be visible, even for strongly underdoped systems. This phenomenon could be used to separate the small contributions due to antiferromagnetic correlations from the dominant, rather conventional, contribution of the $\mathbf{q} \approx 0$ dynamical spin susceptibility. Finally, in a futher step, based on the findings of this paper, it is of interest to investigate the behavior of two coupled, but inequivalent layers in the superconducting state (some work in this direction, although at much higher temperatures, has been carried out in Ref. 19). This may reveal, why the material $\mathrm{Y}_{2} \mathrm{Ba}_{4} \mathrm{Cu}_{7} \mathrm{O}_{15}$ exhibits a higher $\mathrm{T}_{c}$ than both of the corresponding parent compounds it consists of.

This work has been supported in part by the Science and Technology Center for Superconductivity through
NSF-grant DMR91-20000, the Deutsche Forschungsgemeinschaft (J.S.), the EC-TMR program ERBFMBICT950048 (E.A.), and by FORSUPRA (G.H. and W.H.). It is our pleasure to thank H. Monien, D. Pines, R. Stern, M. G. Zacher, and R. Eder for helpful discussions. The calculations were performed at the ZAM in Jülich and the LRZ in Munich.

${ }^{1}$ J. Tranquada, P. Gehring, G. Shirane, S. Shamoto, and M. Sato, Phys. Rev. B 46, 5561 (1992).

${ }^{2}$ R. Stern, M. Mali, I. Mangelschots, J. Roos, and D. Brinkmann, Phys. Rev. B 50, 426 (1994).

${ }^{3}$ R. Stern, M. Mali, J. Roos, and D. Brinkmann, Phys. Rev. B 51, 15478 (1995).

${ }^{4}$ R. Stern, M. Mali, J. Roos, and D. Brinkmann, Phys. Rev. B 52, R15734 (1995).

${ }^{5}$ H. Monien and T. M. Rice, Physica C 235-240, 1705 (1994).

${ }^{6}$ See, e. g., D. K. Morr, A. V. Chubukov, A. P. Kampf, G. Blumberg, cond-mat/9512131, and references therein.

${ }^{7}$ P. Bourges, H. F. Fong, L. P. Regnault, J. Bossy, C. Vettier, D. L. Milius, I. A. Aksay, and B. Keimer, Phys. Rev. B 56, R11 439 (1997).

${ }^{8}$ M. Schabel, C.-H. Park, A. Matsuura, Z.-X. Shen, D. Bonn, R. Liang, and W. Hardy, Phys. Rev. B 57, 6090 (1998).

${ }^{9}$ M. Schabel, C.-H. Park, A. Matsuura, Z.-X. Shen, D. Bonn, R. Liang, and W. Hardy, Phys. Rev. B 57, 6107 (1998).

${ }^{10}$ P. W. Anderson, Science 279, 1196 (1998).

11 A. J. Leggett, Science 279, 1157 (1998).

12 N. Bulut, D. J. Scalapino, and R. T. Scalettar, Phys. Rev. B 45, 5577 (1992).

${ }^{13}$ N. Bulut and D. Scalapino, Phys. Rev. B 53, 5149 (1996).

${ }^{14}$ R. Hetzel, W. v.d. Linden, and W. Hanke, Phys. Rev. B 50, 4159 (1994).

15 T. Dahm and L. Tewordt, Physica C 253, 334 (1995).

16 T. Dahm, D. Manske, and L. Tewordt, Phys. Rev. B 54, 6640 (1996).

${ }^{17}$ S. Grabowski, J. Schmalian, M. Langer, and K. Bennemann, Phys. Rev. B 55, 2784 (1997).

18 S. Grabowski, J. Schmalian, and K. Bennemann, Physica B 230, 948 (1997).

${ }^{19}$ R. T. Scalettar, J. W. Cannon, D. J. Scalapino, and R. L. Sugar, Phys. Rev. B 50, 13419 (1994).

${ }^{20}$ A. J. Millis and H. Monien, Phys. Rev. B 54, 16172 (1996).

${ }^{21}$ N. E. Bickers and D. J. Scalapino, Ann. Phys. 193, 206 (1989).

22 J. Schmalian, M. Langer, S. Grabowski, and K. H. Bennemann, Comp. Phys. Comm. 93, 141 (1996).

${ }^{23}$ D. G. Clarke and S. P. Strong, Adv. Phys. 46, 545 (1997).

${ }^{24}$ H. Monien, N. Elstner, and A. J. Millis, cond-mat/9707051.

${ }^{25}$ E. Arrigoni, Phys. Rev. Lett. 80, 790 (1998).

${ }^{26}$ G. Hildebrand, E. Arrigoni, C. Gröber, and W. Hanke, cond-mat/9801181.

27 B. Shastry, Phys. Rev. Lett. 63, 1288 (1989).

${ }^{28}$ F. Mila and T. Rice, Physica C 157, 561 (1989).

${ }^{29}$ M. Takigawa, Phys. Rev. B 49, 4158 (1994).

${ }^{30}$ D. Thelen and D. Pines, Phys. Rev. B 49, 3528 (1994).

${ }^{31}$ V. Barzykin and D. Pines, Phys. Rev. B 52, 13585 (1995). 